\title{
PROCESSES DISJOINT FROM WEAK MIXING
}

\author{
S. GLASNER AND B. WEISS
}

Dedicated to the memory of Douglas McMahon

\begin{abstract}
We show that the family $\mathscr{W}^{\perp}$ of ergodic measure preserving transformations which are disjoint from every weakly mixing m.p.t. properly contains the family $\mathscr{D}$ of distal ergodic m.p.t. In the topological case we show that $\mathscr{P} \mathscr{F}$, the family of proximally isometric flows is properly contained in the family $\mathscr{M}\left(\mathscr{W}^{\perp}\right)$ of multipliers for $\mathscr{W}^{\perp}$.
\end{abstract}

\section{INTRODUCTION}

Let $\mathscr{W}$ be the class of all weakly mixing measure preserving transformations. $\mathscr{W}^{\perp}$ will then denote the class of all ergodic m.p.t. disjoint from each element of $\mathscr{W}$. It was shown in [F] that $\mathscr{K} \subset \mathscr{W}^{\perp}$ where $\mathscr{K}$ is the family of ergodic Kronecker m.p.t. Since group extensions preserve disjointness, it follows that the class $\mathscr{D}$ of measure theoretically distal, ergodic m.p.t. is also a subset of $\mathscr{W}^{\perp}$. ( $\mathscr{D}=$ ergodic $\mathbb{Z}$-actions with generalized discrete spectrum in the terminology of $[Z]$.) We say that an m.p.t. $(X, \mathscr{F}, \mu, T)$ is a multiplier for $\mathscr{W}^{\perp}$ if for every ergodic m.p.t. $(Y, \mathscr{B}, \nu, S)$ in $\mathscr{W}^{\perp}$, every ergodic joining $(X \times Y, \mathscr{F} \otimes \mathscr{B}, \lambda, T \times S)$ of $\mu$ and $\nu$ is in $\mathscr{W}^{\perp}$. The questions we ask here are

(i) Is $\mathscr{W}^{\perp}=\mathscr{D}$ ?

(ii) Does $\mathscr{M}\left(\mathscr{W}^{\perp}\right)$, the class of ergodic multipliers for $\mathscr{W}^{\perp}$, coincide with $\mathscr{D}$ ?

In $\left[G_{1}\right]$ the first question-in the topological dynamical setting-was investigated. It was shown that the class $\mathscr{P} \mathscr{I}$ of proximally isometric minimal flows satisfies $\mathscr{P} \mathscr{J} \varsubsetneqq \mathscr{W}^{\perp}$, where here $\mathscr{W}$ is the class of topologically weakly mixing minimal flows. (Since for minimal flows disjointness from a minimal topologically weakly mixing flow is preserved by proximal as well as group extensions, $\mathscr{P} \mathscr{F}$ which of course contains the family of minimal distal flows, is the correct substitute for $\mathscr{D}$.)

In this paper we show that in the measure theoretical case $\mathscr{D} \varsubsetneqq \mathscr{W}^{\perp}$. The method used is that of [G-W]. Specifically, we use an irrational rotation $R_{\alpha}$ of

Received by the editors June 15, 1987 and, in revised form, May 20, 1988.

1980 Mathematics Subject Classification (1985 Revision). Primary 28D05; Secondary 54H20. 
the circle as a basis for building, generically skew product extensions with the property of being disjoint from every ergodic m.p.t. which is disjoint from $R_{\alpha}$. When the "fiber flow" is weakly mixing, generically, these extensions will also be weakly mixing extensions of $R_{\alpha}$, hence not in $\mathscr{D}$.

It follows by induction on towers that $\mathscr{D} \subset \mathscr{M}\left(\mathscr{W}^{\perp}\right)\left(\mathscr{P} \mathscr{I} \subset \mathscr{M}\left(\mathscr{W}^{\perp}\right)\right.$ in the topological case). Do we have equalities? We use a slight generalization of a result of D. MacMahon to show that $\mathscr{P} \mathscr{F} \varsubsetneqq \mathscr{M}\left(\mathscr{W}^{\perp}\right)$. The corresponding question remains open in the measure theoretical case.

In $\S 1$ we show that generically skew extensions of a strictly ergodic flow $(Z, \sigma)$ with a weakly mixing real fiber flow $(Y, \mathbb{R})$ yield weakly mixing extensions.

In $\S 2$ we prove the existence of an ergodic m.p.t. in $\mathscr{W}^{\perp} \backslash \mathscr{D}$. The proof of a key lemma whose proof is probabilistic is postponed to $\S 3$. In $\S 4$ we show that in the topological case $\mathscr{P} \mathscr{I} \varsubsetneqq \mathscr{M}\left(\mathscr{W}^{\perp}\right)$.

\section{WEAK MIXING EXTENSIONS}

Let $(Z, \sigma)$ be a strictly ergodic flow with a unique invariant measure $\mu$. Let $\left(Y,\left\{h_{t}\right\}_{t \in \mathbb{R}}\right)=(Y, \mathbb{R})$ be a real flow preserving a measure $\nu$ such that the measure preserving real flow $(Y, \nu, \mathbb{R})$ is weakly mixing. Put $X=Z \times Y$ and let $X \stackrel{\pi}{\rightarrow} Z$ be the projection map. Denote the group of homeomorphisms of $Y$ by $\mathscr{H}(Y)$ and denote the subgroup $\left\{h_{t}\right\}_{t \in \mathbb{R}}$ by $\mathscr{G}$. With each continuous map $z \rightarrow g_{z}$ of $Z$ into $\mathscr{G}$ we associate the homeomorphism $G$ of $X$ defined by $G(z, y)=\left(z, g_{z}(y)\right)$. Let $\mathscr{G}_{s}$ be the subgroup of $\mathscr{H}(X)$ which consists of all such $G$. Put

$$
\mathscr{S}_{\mathscr{G}}(\sigma)=\left\{G^{-1} \circ(\sigma \times \mathrm{id}) \circ G: G \in \mathscr{G}_{s}\right\} \text {. }
$$

We use the topology of uniform convergence of homeomorphisms and their inverses on $\mathscr{H}(X)$. Under this topology $\mathscr{H}(X)$ becomes a complete metrizable space.

1.1 Theorem. There exists a residual subset $\mathscr{R} \subset \overline{\mathscr{S}_{\mathscr{G}}(\sigma)}$ such that $T \in \mathscr{R}$ implies $(X, T, \mu \times \nu) \stackrel{\pi}{\rightarrow}(X, \sigma, \mu)$ is a weakly mixing extension.

Proof. By definition $\pi$ is w.m. w.r.t. $T$ if the map induced by $T$ on $(Z \times$ $Y \times Y, \mu \times \nu \times \nu)$ is ergodic. For a continuous function $f$ on $Z \times Y \times Y$ with $\int f d \nu \times \nu \times \nu=0, \int|f|^{2} d \mu \times \nu \times \nu=1$, and $\varepsilon>0$, put

$$
E_{f, \varepsilon}=\left\{T \in \overline{\mathscr{S}_{\mathscr{f}}(\sigma)}: \exists n \text { with }\left|\frac{1}{n} \sum_{j=0}^{n-1} \int\left(f \circ T^{k}\right) \cdot f d \mu \times \nu \times \nu\right|<\varepsilon\right\} .
$$

Then choosing an appropriate sequence of such functions $\left\{f_{i}\right\}_{i=1}^{\infty}$ the set $\mathscr{R}=$ $\bigcap_{i, j} E_{f_{i}, 1 / j}$ will be the desired set, provided we show that each of the open sets $E_{f, \varepsilon}$ is dense in $\overline{\mathscr{S}}_{\tilde{r},(\sigma)}$.

Now given $G \in \mathscr{G}_{S}$ we have

$$
G \circ E_{f, \varepsilon} \circ G^{-1}=E_{f \circ G^{-1}, \varepsilon} .
$$


Thus $G^{-1} \circ \sigma \circ G \in \bar{E}_{f, \varepsilon} \Leftrightarrow \sigma \in \bar{E}_{f \circ G^{-1}, \varepsilon}$. (We write $G^{-1} \circ \sigma \circ G$ for $G^{-1} \circ$ $(\sigma \times \mathrm{id}) \circ G$.) Therefore in order to show that each $\bar{E}_{f, e}$ is dense it suffices to show that $\sigma \in \bar{E}_{f, e}$. In turn this will follow if we show the following:

Claim. Given $f$ and $\varepsilon$ as above and $\delta>0$ there exists $G \in \mathscr{G}_{s}$ such that

(i) $d\left(\sigma, G^{-1} \circ \sigma \circ G\right)<\delta$,

(ii) $G^{-1} \circ \sigma \circ G \in E_{f, \varepsilon}$.

We proceed to prove this claim. First consider for any $G \in \mathscr{G}_{S}$ the sums

$$
\begin{aligned}
& \frac{1}{n} \sum_{j=0}^{n-1} \int\left(f \circ G^{-1} \circ \sigma^{k} \circ G\right) \cdot f d \mu \times \nu \times \nu \\
& =\frac{1}{n} \sum_{j=0}^{n-1} \int f\left(\sigma^{k} z, g_{\sigma^{k} z}^{-1} g_{z}\left(y_{1}\right), g_{\sigma^{k} z}^{-1} g_{z}\left(y_{2}\right)\right) \\
& \cdot f\left(z, y_{1}, y_{2}\right) d \mu(z) d \nu\left(y_{1}\right) d \nu\left(y_{2}\right) .
\end{aligned}
$$

Interchanging sum and integral signs we get by strict ergodicity of $(Z, \sigma)$ that these sums tend uniformly in $z, y_{1}, y_{2}$ to

$$
\iiint \int f\left(w, g_{w}^{-1} g_{z}\left(y_{1}\right), g_{w}^{-1} g_{z}\left(y_{2}\right)\right) f\left(z, y_{1}, y_{2}\right) d \mu(w) d \mu(z) d \nu\left(y_{1}\right) d \nu\left(y_{2}\right) \text {. }
$$

Thus in order to show (ii) it suffices to show that $G$ can be chosen so that the absolute value of the last expression is smaller than $\varepsilon$. This will be done by showing that already the expression

$$
\int f\left(w, g_{w}^{-1} g_{z}\left(y_{1}\right), g_{w}^{-1} g_{z}\left(y_{2}\right)\right) d \mu(w)
$$

is small for most $\left(y_{1}, y_{2}\right)$. In turn this will be done by showing that the integral in $(*)$ is small over very small sets where the dependence of $f$ on the first variable can be neglected.

The idea of the construction of $G$ is roughly this. We split (almost all of) $Z$ into finitely many Cantor sets $K_{i j}$, on each of which $f\left(w, y_{1}, y_{2}\right)$ depends only slightly on $w \in K_{i j}$. Choosing $w_{i j} \in K_{i j}$ we let $f_{i j}\left(y_{1}, y_{2}\right)=f\left(w_{i j}, y_{1}, y_{2}\right)$. These are continuous functions on $Y \times Y$ and we use the weak mixing of $(Y, \mathbb{R})$ to find an $L>0$ for which $\frac{1}{L} \int_{0}^{L} f_{i j}\left(h_{t} y_{1}, h_{t} y_{2}\right) d t$ approximately equals $\iint f_{i j}(u, v) d \nu(u) d \nu(v)$ for $\left(y_{1}, y_{2}\right)$ in a big subset of $Y \times Y$. We then define $\theta: K_{i j} \rightarrow[0, L]$ so that $\theta\left(\left(\mu \mid K_{i j}\right) / \mu\left(K_{i j}\right)\right)$ is normalized Lebesgue measure on $[0, L]$. Now $\theta$ is extended to all of $Z$ and we define $g_{z}=h_{\theta(z)}^{-1}$. These considerations and constructions put together should yield (ii). Property (i) is obtained by letting $A=\bigcup_{j=1}^{M} K_{0 j}$ be a basis for a Rohlin tower $A, \sigma A, \ldots, \sigma^{N^{2}-1} A$ (large $N$ ); (thus $K_{i j}=\sigma^{i} K_{0 j}$ ). Defining $\theta$ in such a way that $\theta(\sigma z)=\theta(z)$ for $z \in \bigcup_{i=1}^{N^{2}-N-1} \sigma^{i} A$ we make $G$ almost commute with $\sigma$. We now make these ideas precise. 
By weak mixing of $(Y, \mathbb{R}, \nu)$ and continuity of $f$ there exists an $L>0$ and a set $W \subset Y \times Y$ with $\nu \times \nu(W)>1-\varepsilon$ such that $\left(y_{1}, y_{2}\right) \in W$ implies

$$
\forall z \in Z, \quad\left|\frac{1}{L} \int_{0}^{L} f\left(z, h_{t} y_{1}, h_{t} y_{2}\right) d t-\int f(z, u, v) d \nu(u) d \nu(v)\right|<\varepsilon .
$$

Fix $\alpha>0$ such that $d\left(z_{1}, z_{2}\right)<\alpha$ implies $\left|f\left(z_{1}, y, y^{\prime}\right)-f\left(z_{2}, y, y^{\prime}\right)\right|<\varepsilon$ for all $y, y^{\prime} \in Y$. Let $\eta>0$ be such that $\left|t_{1}-t_{2}\right|<\eta$ implies $d\left(h_{t_{1}}^{-1} h_{t_{2}}\right.$, id $)<\delta$ and choose $N \in \mathbb{N}$ for which $\frac{2 L}{N}<\eta$ and $\frac{1}{N}<\varepsilon$.

By Rohlin's lemma there exists a Cantor (i.e., closed totally disconnected perfect) subset $A$ of $Z$ with $\mu(A)>0$, such that $A, \sigma A, \ldots, \sigma^{N^{2}-1} A$ are pointwise disjoint and such that $1-\mu\left(\bigcup_{i=0}^{N^{2}-1} \sigma^{i} A\right)<\varepsilon$. We can further split $A$ into a disjoint union $A=\bigcup_{j=1}^{M} K_{j}$ of Cantor sets $K_{j}$ such that $\mu\left(K_{j}\right)>0$ and radius $\left(\sigma^{i} K_{j}\right)<\alpha$ for $1 \leq j \leq M, 0 \leq i \leq N^{2}-1$. Finally choose points $w_{j} \in K_{j}$ and define

We let

$$
f_{i j}\left(y_{1}, y_{2}\right)=f\left(\sigma^{i} w_{j}, y_{1}, y_{2}\right) \text {. }
$$

$$
K=\bigcup_{i=0}^{N^{2}-1} \bigcup_{j=1}^{M} \sigma^{i}\left(K_{j}\right)=\bigcup_{i=0}^{N^{2}-1} \sigma^{i} A
$$

We have for all $z, y_{1}, y_{2}$

$$
\begin{aligned}
& \mid \int f\left(w, g_{w}^{-1} g_{z}\left(y_{1}\right), g_{w}^{-1} g_{z}\left(y_{2}\right)\right) f\left(z, y_{1}, y_{2}\right) d \mu(w) \\
& \quad-\int 1_{K}(w) f\left(w, g_{w}^{-1} g_{z}\left(y_{1}\right), g_{w}^{-1} g_{z}\left(y_{2}\right)\right) f\left(z, y_{1}, y_{2}\right) d \mu(w) \mid=O(\varepsilon)
\end{aligned}
$$

and

$$
\begin{aligned}
& \mid \int 1_{K}(w) \cdot f\left(w, g_{w}^{-1} g_{z}\left(y_{1}\right), g_{w}^{-1} g_{z}\left(y_{2}\right)\right) f\left(z, y_{1}, y_{2}\right) d \mu(w) \\
& \quad-\sum_{i j} \int 1_{\sigma^{i} K_{j}}(w) f_{i j}\left(g_{w}^{-1} g_{z}\left(y_{1}\right), g_{w}^{-1} g_{z}\left(y_{2}\right)\right) f\left(z, y_{1}, y_{2}\right) d \mu(w) \mid=O(\varepsilon) .
\end{aligned}
$$

For $\left(y_{1}, y_{2}\right) \in W$ we have

$$
\left|\frac{1}{L} \sum_{i j} \mu\left(K_{j}\right) \int_{0}^{L} f_{i j}\left(h_{t} y_{1}, h_{t} y_{2}\right) d t-\sum_{i j} \mu\left(K_{j}\right) \iint f_{i j}(u, v) d \nu(u) d \nu(v)\right|<\varepsilon .
$$

For each $j$ let $\tilde{\theta}: K_{j} \rightarrow[0, L]$ be a continuous map for which $\tilde{\theta}\left(\mu \mid K_{j} / \mu\left(K_{j}\right)\right)$ $=\lambda / L$ where $\lambda$ is Lebesgue measure on $[0, L]$.

Define $\tilde{\theta}$ on $K=\bigcup_{j=1}^{M} \bigcup_{i=0}^{N^{2}-1} \sigma^{i} K_{j}$ by $\tilde{\theta}(z)=\tilde{\theta}\left(\sigma^{-i} z\right)$ if $z \in \sigma^{i} K_{j}$, and extend it to a continuous map $\tilde{\theta}: Z \rightarrow[0, L]$. Put $\theta(z)=\frac{1}{N} \sum_{i=0}^{N-1} \tilde{\theta}\left(\sigma^{i} z\right)$ and $g_{z}=h_{\theta(z)}^{-1}$. 
We now have

$$
\begin{aligned}
& \mid \sum_{i j} \int 1_{K}(w) f_{i j}\left(g_{w}^{-1} g_{z}\left(y_{1}\right), g_{w}^{-1} g_{z}\left(y_{2}\right)\right) f\left(z, y_{1}, y_{2}\right) d \mu(w) \\
& \quad-\sum_{i=1}^{N^{2}-N-1} \sum_{j=1}^{M} \int_{K_{j}} f_{i j}\left(h_{\theta\left(\sigma^{i} w\right)} g_{z}\left(y_{1}\right), h_{\theta\left(\sigma^{i} w\right)} g_{z}\left(y_{2}\right)\right) f\left(z, y_{1}, y_{2}\right) d \mu(w) \mid \\
& \quad=O(\varepsilon) .
\end{aligned}
$$

But for $w \in \bigcup_{j=1}^{M} K_{j}, 0 \leq i \leq N^{2}-N-1$, and $z, y_{1}, y_{2}$ with

$$
\left(g_{z}\left(y_{1}\right), g_{z}\left(y_{2}\right)\right) \in W, \quad \theta\left(\sigma^{i} w\right)=\tilde{\theta}(w)
$$

and

$$
\begin{array}{r}
\int_{K_{j}} f_{i j}\left(h_{\tilde{\theta}\left(\sigma^{i} w\right)} g_{z}\left(y_{1}\right), h_{\tilde{\theta}\left(\sigma^{i} w\right)} g_{z}\left(y_{2}\right)\right) f\left(z, y_{1}, y_{2}\right) d \mu(w) \\
=\frac{\mu\left(K_{j}\right)}{L} \int_{0}^{L} f_{i j}\left(h_{t} g_{z}\left(y_{1}\right), h_{t} g_{z}\left(y_{2}\right)\right) f\left(z, y_{1}, y_{2}\right) d t
\end{array}
$$

Collecting our estimates we get for $\left(g_{z}\left(y_{1}\right), g_{z}\left(y_{2}\right)\right) \in W$

$$
\begin{aligned}
& \mid \int f\left(w, g_{w}^{-1} g_{z}\left(y_{1}\right), g_{w}^{-1} g_{z}\left(y_{2}\right)\right) f\left(z, y_{1}, y_{2}\right) d \mu(w) \\
& \quad-\sum_{i j} \mu\left(K_{j}\right) \iint f_{i j}(u, v) f\left(z, y_{1}, y_{2}\right) d \nu(u) d \nu(v) \mid=O(\varepsilon) .
\end{aligned}
$$

Since also

$$
\left|\sum_{i, j} \mu\left(K_{j}\right) \iint f_{i j}(u, v) d \nu(u) d \nu(v)-\int f d \mu \times \nu \times \nu\right|=O(\varepsilon)
$$

and since $\int f d \mu \times \nu \times \nu=0$ we get

$$
\left|\int f\left(w, g_{w}^{-1} g_{z}\left(y_{1}\right), g_{w}^{-1} g_{z}\left(y_{2}\right)\right) f\left(z, y_{1}, y_{2}\right) d \mu(w)\right|=O(\varepsilon) .
$$

For a fixed $z \in Z \quad g_{z}$ preserves $\nu$. Since $\nu_{1} \times \nu_{2}(W)>1-\varepsilon$ we get

$$
\left|\iiint f\left(w, g_{w}^{-1} g_{z}\left(y_{1}\right), g_{w}^{-1} g_{z}(y)\right) f\left(z, y_{1}, y-2\right) d \mu(w) d \nu\left(y_{1}\right) d \nu\left(y_{2}\right)\right|=O(\varepsilon) \text {. }
$$

Finally we integrate w.r.t. $d \mu(z)$ and obtain (ii) of our claim.

(i) follows since for each $z \in Z \quad|\theta(\sigma z)-\theta(z)|<\frac{2 L}{N}<\eta$. Hence

$$
d\left(g_{\sigma z}^{-1} g_{z}, \mathrm{id}\right)=d\left(h_{\theta(\sigma z)} h_{\theta(z)}^{-1}, \mathrm{id}\right)<\delta .
$$

Our proof is complete. 


\section{2. $\mathscr{D} \varsubsetneqq \mathscr{W}^{\perp}$}

When working in the measure theoretical category we restrict our discussion to quadruples $(X, \mathscr{B}, \mu, T)$ where $X$ is compact metric, $\mathscr{B}$ its Borel field, $\mu$ a probability measure and $T$ a self-homeomorphism of $X$. We call such an object a topological process. To see that this is justified we refer the reader to, e.g., $\left[G_{2}\right]$. When $X$ is a topological space, $\mathscr{P}(X)$ denotes the space of probability measures on $X$ with the weak $*$ topology. For two topological processes $\mathscr{X}=(X, \mathscr{B}, \mu, T)$ and $\mathscr{Y}=(Y, \mathscr{F}, \nu, S)$ a joining is a $T \times S$ invariant measure $\lambda$ on $X \times Y$ such that $\pi_{X} \lambda=\mu$ and $\pi_{Y} \lambda=\nu . \mathscr{X}$ and $\mathscr{Y}$ are disjoint if their only ergodic joining is $\lambda=\mu \times \nu$. A flow is a pair $(X, T)$ where $X$ is a compact metric space and $T$ a homeomorphism of $X$; or more generally a pair $(X, \mathscr{G})$ where $\mathscr{G}$ is a group of homeomorphisms of $X$.

2.1 Proposition. Let $(X, \eta, T) \stackrel{\pi}{\rightarrow}(Z, \mu, T)$ be a homomorphism of strictly ergodic flows. Let $\mathscr{M}=\{\theta \in \mathscr{P}(X): \pi(\theta)=\mu\}$, then $\mathscr{M}$ is T-invariant. Suppose $\delta_{\eta}$ (the point mass at $\left.\eta \in \mathscr{P}(X)\right)$ is the unique T-invariant measure on $\mathscr{M}$, then $(X, \eta, T)$ is measure theoretically disjoint from every ergodic topological process which is disjoint from $(Z, \mu, T)$.

Proof. Let $(W, \nu, S)$ be ergodic and disjoint from $(Z, \mu, T)$. Let $\lambda \in$ $\mathscr{P}(W \times X)$ be an ergodic joining of $\nu$ and $\eta$ and let

$$
\lambda=\int \delta_{w} \times \lambda_{w} d \nu(w)
$$

be a disintegration of $\lambda$ with respect to $\nu$. Projecting on $W \times X$ we get a joining $\lambda^{\prime}$ of $\nu$ and $\mu$ with the corresponding disintegration

$$
\lambda^{\prime}=\int \delta_{w} \times \lambda_{w}^{\prime} d \mu(w) .
$$

Since $\nu$ and $\mu$ are disjoint $\lambda^{\prime}=\nu \times \mu=\int \delta_{w} \times \mu d \nu(w)$. By uniqueness of distintegraton we conclude that for $\nu$-a.e. $w \quad \lambda_{w}^{\prime}=\mu$. Thus $\pi\left(\lambda_{w}\right)=\lambda_{w}^{\prime}=\mu$ and therefore $\lambda_{w} \in \mathscr{M}$ for $\nu$-a.e. $w \in W$. The map $w \rightarrow \lambda_{w}$ is measurable and carries $\nu$ onto a $T$-invariant ergodic measure $\nu^{*}$ on $\mathscr{M}$. (In the terminology of $\left[G_{2}\right]\left(\mathscr{M}, \nu^{*}, T\right)$ is a quasi-factor of $(X, \eta, T)$.) However, by assumption, the only $T$-invariant measure on $\mathscr{M}$ is $\delta_{\eta}$ so that $\nu^{*}=$ $\delta_{\eta}$ and $\lambda_{w}=\eta$ for $\nu$-a.e. $w$. This concludes the proof since now $\lambda=$ $\int \delta_{w} \times \lambda_{w} d \nu(w)=\nu \times \eta$.

Let $(Z, \sigma)$ be an irrational rotation of the circle. $(Z=\mathbf{T}=[0,1), \sigma z=$ $z+\alpha \bmod 1, \alpha \in \mathbb{R} \backslash Q)$. Let $\left(Y,\left\{h_{t}\right\}_{t \in \mathbb{R}}\right)=(Y, \mathbb{R})$ be a strictly ergodic real flow with invariant measure $\nu$. Put $X=Z \times Y$ and let $X \stackrel{\pi}{\rightarrow} Z$ be the projection map. We denote Lebesgue measure on $Z$ by $\mu$. Denote the group of homeomorphisms of $Y$ by $\mathscr{H}(Y)$ and denote the subgroup $\left\{h_{t}\right\}_{t \in \mathbb{R}}$ by $\mathscr{G}$. With each continuous map $z \rightarrow g_{z}$ of $Z$ into $\mathscr{G}$ we associate the homeomorphism $G$ of $X$ defined by $G(z, y)=\left(z, g_{z}(y)\right)$. Let $\mathscr{G}_{\mathscr{Y}}$ be a subgroup of 
$\mathscr{H}(X)$ which consists of all such $G$. Put

$$
\mathscr{S}_{\mathscr{G}}(\sigma)=\left\{G^{-1} \circ(\sigma \times \mathrm{id}) \circ G: G \in \mathscr{G}_{\mathscr{S}}\right\}
$$

We use the topology of uniform convergence of homeomorphisms and their inverses on $\mathscr{H}(X)$.

2.2 Theorem. There exists a residual subset $\mathscr{R} \subset \overline{\mathscr{S}_{\mathscr{G}}(\sigma)}$ such that $T \in \mathscr{R}$ implies $(X, \mu \times \nu, T)$ is in $\{(Z, \sigma)\}^{\perp \perp}$, hence also in $\mathscr{W}^{\perp}$.

Proof. We split the proof into several steps.

1. Set $\mathscr{M}=\{\theta \in \mathscr{P}(X): \pi(\theta)=\mu\}$; clearly $\mathscr{M}$ is $T$-invariant for every $T \in \overline{\mathscr{S}_{\mathscr{G}}(\sigma)}$. By Proposition 2.1 it is enough to show the existence of a residual $\mathscr{R} \subset \widetilde{\mathscr{S}_{\mathscr{G}}(\sigma)}$ such that $T \in \mathscr{R}$ implies the unique ergodicity of $(\mathscr{M}, T)$ with $\delta_{\mu \times \nu}$ as the unique invariant measure. (Note that this implies in particular that $(X, T, \mu \times \nu)$ is strictly ergodic.)

2. Let $V$ be a neighborhood of $\mu \times \nu$ in $\mathscr{M}$. Put

$$
E_{V, K_{0}, \delta}=\left\{T \in \overline{\mathscr{S}_{\mathscr{G}}(\sigma)}: \exists K \geq K_{0} \text { such that } \forall \theta \in \mathscr{M}\right. \text { at most }
$$

$[K \delta]$ elements of the set $\left\{\theta, T \theta, \ldots, T^{K} \theta\right\}$ are not in $\left.V\right\}$.

Then $E_{V, K_{0}, \delta}$ is open in $\overline{\mathscr{S}_{\mathscr{G}}(\sigma)}$ and by looking at generic points $\theta$ for ergodic invariant measures on $\mathscr{P}(\mathscr{M})$ we see that for a sequence $V_{K} \searrow\{\mu \times \nu\}$, $\mathscr{R}=\bigcap E_{V_{K}, K, 1 / K}$ is the desired set, provided that for each $V, K$ and $\delta$, $E_{V, K, \delta}$ is dense in $\overline{\mathscr{S}_{\mathscr{G}}(\sigma)}$.

3. By the nature of the topology on $\mathscr{M}$ it is enough to consider neighborhoods $V$ of the form $V=V_{f, \varepsilon}=\left\{\theta \in \mathscr{M}:\left|\int f d \theta\right|<\varepsilon\right\}$ where $f \in C(X), \int f d \mu \times$ $\nu=0$ and $\|f\|_{\infty} \leq 1$. Now for $G \in \mathscr{G}_{\mathscr{S}}$ we have $G E_{V, K, \delta} G^{-1}=E_{V^{\prime}, K, \delta}$ where $V^{\prime}=V_{f \circ G, \varepsilon}$; therefore $G^{-1} \circ \sigma \circ G \in \bar{E}_{V, K, \delta} \Leftrightarrow \sigma \in G \bar{E}_{V, K, \delta} G^{-1} \Leftrightarrow \sigma \in$ $\bar{E}_{V^{\prime}, K, \delta}$. Thus to show that $E_{V, K, \delta}$ is dense it suffices to show that for each $V=V_{f, \varepsilon}$ and $K, \sigma \in \bar{E}_{V, K, \varepsilon}$. For this the following will suffice:

Claim. Given $\varepsilon, \delta^{\prime}>0$ and $K$, there exists $G \in \mathscr{G}_{\mathscr{P}}$ such that

(i) $d\left(\sigma, G^{-1} \circ \sigma \circ G\right)<\delta^{\prime}$,

(ii) $G^{-1} \circ \sigma \circ G \in E_{V, K, \varepsilon}$.

4. To get (ii) of the claim in step 3 it suffices to find $G$ for which

$$
\operatorname{Sup}_{\theta \in \mathscr{M}} \frac{1}{K+1} \sum_{k=0}^{K}\left|\int f d\left(G^{-1} \circ \sigma^{k} \circ G(\theta)\right)\right|^{2}<\varepsilon^{2} .
$$


5. Write

$$
\begin{aligned}
& \frac{1}{K+1} \sum_{k=0}^{K}\left|\int f d\left(G^{-1} \circ \sigma^{k} \circ G(\theta)\right)\right|^{2} \\
& =\frac{1}{K+1} \sum_{k=0}^{K} \iint f\left(\sigma^{k} z_{0}, g_{\sigma^{k} z_{0}}^{-1} g_{z_{0}}(y)\right) \\
& \cdot f\left(\sigma^{k} w_{0}, g_{\sigma^{k} w_{0}} g_{w_{0}}(v)\right) d \theta\left(w_{0}, v\right) d \theta\left(z_{0}, y\right) .
\end{aligned}
$$

For fixed $z_{0}$ and $w_{0}=z_{0}+t$ the sums

$$
\frac{1}{k+1} \sum_{k=0}^{K} f\left(\sigma^{k} z_{0}, g_{\sigma^{k} z_{0}}^{-1} g_{z_{0}}(y)\right) f\left(\sigma^{k}\left(z_{0}+t\right), g_{\sigma^{k}\left(z_{0}+t\right)}^{-1} g_{z_{0}+t}(v)\right)
$$

converge by strict ergodicity of $(Z, \sigma)$, uniformly in $t, y$ and $v$ to

$$
\int f\left(z, g_{z}^{-1} g_{z_{0}}(y)\right) f\left(z+t, g_{z+t}^{-1} g_{z_{0}+t}(v)\right) d \mu(z) \text {. }
$$

Interchanging sum and integration we therefore have

$$
\frac{1}{K+1} \sum_{k=0}^{K}\left|\int f d\left(G^{-1} \circ \sigma^{k} \circ G(\theta)\right)\right|^{2} \rightarrow I
$$

where

$$
I=\iiint f\left(\left(z, g_{z}^{-1} g_{z_{0}}(y)\right) f\left(z+t, g_{z+t}^{-1} g_{z_{0}+t}(v)\right) d \mu(z) d \theta\left(z_{0}+t, v\right) d \theta\left(z_{0}, y\right)\right. \text {. }
$$

6. Write $F(z)=\int f(z, y) d \nu(y)$ then our assumption $\int f d \mu \times \nu=0$ implies

$$
\iint F(z) F(w) d \mu(z) d \mu(w)=0 \text {. }
$$

Since $f$ is uniformly continuous there exists $N$ such that

(i) $|z-j / N| \leq 2 / N \Rightarrow|f(j / N, y)-f(z, y)|<\varepsilon^{2} \quad \forall y \in Y$ and

(ii) $\left|\left(1 / N^{2}\right) \sum_{j, k=0}^{N-1} F(j / N) F(k / N)\right|<\varepsilon^{2}$.

For $t \in[0,1)$ we let $k(t)=k$ if $\frac{k}{N} \leq t<\frac{k+1}{N}$. Coming back to integral $I$ of step 5 , we write

$$
\begin{aligned}
I=\sum_{j=0}^{N-1} \iiint f\left(z, g_{z}^{-1} g_{z_{0}}(y)\right) & f\left(z+t, g_{z+t}^{-1} g_{z_{0}+t}(v)\right) \\
\cdot & 1_{[j / N, j+1 / N]}(z) d \mu(z) d \theta\left(z_{0}+t, v\right) d \theta\left(z_{0}, y\right) .
\end{aligned}
$$

By (i) above we have for each $j$ and $t$ :

$$
\begin{aligned}
& \mid \int_{j / N}^{j+1 / N} f\left(z, g_{z}^{-1} g_{z}(y)\right) f\left(z+t, g_{z+t}^{-1} g_{z_{0}+t}(v)\right) d \mu(z) \\
& \quad-\int_{j / N}^{j+1 / N} f\left(\frac{j}{N}, g_{z}^{-1} g_{z_{0}}(y)\right) f\left(\frac{j+k(t)}{N}, g_{z+t} g_{z_{0}+t}(v)\right) d \mu(z) \mid<\frac{2 \varepsilon^{2}}{N} .
\end{aligned}
$$


2.3 Lemma. Given $f_{1}, f_{2}, \ldots, f_{N}$ continuous functions on $Y$ and $\varepsilon, \delta>0$ there exists a continuous map $\varphi:[0,1] \rightarrow \mathbb{R}$ and a measurable set $D \subset[0,1]$ such that

(i) $\forall s \in[0,1],|\varphi(s+\alpha)-\varphi(s)|<\delta \quad(s+\alpha$ is taken $\bmod 1)$.

(ii) $\forall u, v \in Y$ and $t \in D$ and $1 \leq j \leq N, 1 \leq i, k \leq N$

$$
\left|N \int_{j / N}^{j+1 / N} f_{i}\left(h_{\varphi(z)}(u)\right) f_{k}\left(h_{\varphi(z+t)}(v)\right) d \mu(z)-F_{i} F_{k}\right|<\varepsilon
$$

where $F_{i}=\int f_{i}(y) d \nu(y)$.

(iii) $\mu(D)>1-\varepsilon$.

We defer the proof of this lemma to the next section.

8. We now apply Lemma 2.3 to the functions $f_{j}(y)=f\left(\frac{j-1}{N}, y\right), 1 \leq j \leq N$, and define $g_{z}=h_{\varphi(z)}^{-1}, z \in[0,1)$. If $\delta$ is chosen so that $\left\|h_{t}-h_{0}\right\|<\delta^{\prime}$ for $|t|<\delta$ then

$$
d\left(G^{-1} \circ \sigma \circ G, \sigma\right)=\operatorname{Sup}_{(z, y)} d\left(\left(z+\alpha, h_{\varphi(z+\alpha)-\varphi(z)}(y)\right),(z+\alpha, y)\right)<\delta^{\prime}
$$

so that (i) of the claim in step 3 is satisfied.

9. Write $I=I_{1}+I_{2}$ where in $I$, we integrate with respect to $d \theta\left(z_{0}+t, v\right)$ (fixed $z_{0}$ ) over $D \times Y$ and in $I_{2}$ over $([0,1) \backslash D) \times Y=D^{c} \times Y$. Since $\|f\|_{\infty} \leq 1$ we have $\left|I_{2}\right| \leq \mu\left(D^{c}\right)<\varepsilon$. Using Lemma 2.3 we get for $t \in D$ and all $z_{0}, y, v$ and $j$

$$
\begin{array}{r}
\mid \int_{j / N}^{j+1 / N} f\left(\frac{j}{N}, g_{z}^{-1} g_{z_{0}}(y)\right) f\left(\frac{j+k(t)}{N}, g_{z+t}^{-1} g_{z_{0}+t}(v)\right) d \mu(z) \\
-\frac{1}{N} F\left(\frac{j}{N}\right) F\left(\frac{j+k(t)}{N}\right) \mid<\frac{\varepsilon}{N} .
\end{array}
$$

Applying the estimation we obtained at step 6 we now have

$$
I_{1}=\frac{1}{N} \sum_{j=0}^{N-1} \int F\left(\frac{j}{N}\right) F\left(\frac{j+k(t)}{N}\right) d \mu(t)+O(\varepsilon) ;
$$

because $\theta \in \mathscr{M}$, so that integration with respect to $d \theta\left(z_{0}+t, v\right)$ (fixed $z_{0}$ ) of a function which does not depend on $v$ is the same as integration with respect to $d \mu(t)$. From this we obviously have

$$
I_{1}=\frac{1}{N^{2}} \sum_{j, k=0}^{N-1} F\left(\frac{j}{N}\right) F\left(\frac{k}{N}\right)+O(\varepsilon)
$$

and (ii) of step 6 now yields $I_{1}=O(\varepsilon)$. Hence $I=I_{1}+I_{2}=O(\varepsilon)$ and (ii) of the claim in step 3 is also satisfied by our choice of $G$. This completes the proof of Theorem 2.2 .

Remarks. 1. Theorem 2 of [G-W] ensures the existence of a residual set in $\overline{\mathscr{S}_{\mathscr{S}}(\sigma)}$ of strictly ergodic transformations under either one of two strong conditions $A$ or $B$. Condition $B$ is that $\mathscr{G}$ is a compact peano space and that it 
acts transitively on $Y$. Condition $A$ is a rather strong compressibility condition of the flow $(Y, \mathscr{G})$. Theorem 2.1 above implies that under the mild assumption that $(Y, \mathscr{R})$, is strictly ergodic every member of $\mathscr{R}$ is strictly ergodic. In fact if strict ergodicity is all we want then a much easier proof can be given and the conditions on $(Z, \sigma)$ and $(Y, \mathscr{G})\left((Z, \sigma)=\left(\mathbf{T}, R_{\alpha}\right)\right.$ and $(Y, \mathscr{G})$ being a real flow) can be relaxed. (See also $[N]$.)

2. An ergodic process of positive entropy always admits a weakly mixing factor of positive entropy (e.g., a Bernoulli shift). Thus every element of $\mathscr{W}^{\perp}$ is necessarily of zero entropy. In particular a strictly ergodic member of $\mathscr{W}^{\perp}$ has zero topological entropy.

2.4 Theorem. There exists an ergodic measure preserving transformation $(X, \eta$, T) which is in $\mathscr{W}^{\perp}$ but not in $\mathscr{D}$.

Proof. Notations as in Theorem 2.2, we now further assume that $(Y, \mathbb{R})$ is weakly mixing. Then by Theorem 1.1 there exists a residual subset $\mathscr{R}_{1} \subset$ $\overline{\mathscr{S}_{\mathscr{f}}(\sigma)}$ such that $T \in \mathscr{R}_{1}$ implies $(X, \mu \times \nu, T)$ is a weakly mixing extension of $(Z, \mu, \sigma)$. By Proposition 8.6 and Lemma 8.11 of [Z] we conclude that $(X, \mu \times \nu, T) \notin \mathscr{D}$. Our theorem is now valid for each $T$ in $\mathscr{R} \cap \mathscr{R}_{1}$.

\section{THE PROOF OF LEMMA 2.3}

Let us recapitulate our situation here. We have a strictly ergodic real flow $h_{t}: Y \rightarrow Y$ with invariant measure $\nu$. We are given a finite set of continuous functions $f_{1}, \ldots, f_{N}$ on $Y$ and $\varepsilon>0, \delta>0$, and wish to find a continuous map $\varphi: \mathbb{R} / \mathbb{Z} \rightarrow \mathbb{R}$ such that

(1) For a measurable subset $D \subset \mathbb{R} / \mathbb{Z}$ of measure $1-\varepsilon$, and all $t \in D$, all $u, v \in Y$ we have

$$
\begin{aligned}
& \mid N \int_{j / N}^{j+1 / N} f_{i}\left(h_{\varphi(z)}(u)\right) f_{k}\left(h_{\varphi(z+t)}(v)\right) d \mu(z) \\
& \quad-\left(\int f_{i}(y) d \nu(y)\right)\left(\int f_{k}(y) d \nu(y)\right) \mid<\varepsilon
\end{aligned}
$$

for all $1 \leq j \leq N, 1 \leq i, k \leq N$.

(2) $|\varphi(t+\alpha)-\varphi(t)|<\delta$ for all $t \in \mathbb{R} / \mathbb{Z}$.

The idea of the construction is as follows. The strict ergodicity of the flow means that for any $\gamma>0$ there is some $T_{0}$ sufficiently large so that for all $T \geq T_{0}$, uniformly in $u \in Y$

$$
\left|\frac{1}{T} \int_{0}^{T} f_{i}\left(h_{s}(u)\right) d s-\int_{Y} f_{i}(y) d \nu(y)\right|<\gamma
$$

for $1 \leq i \leq N$. It follows that

$$
\left|\frac{1}{T^{2}} \int_{0}^{T} \int_{0}^{T} f_{i}\left(h_{r}(u)\right) f_{k}\left(h_{s}(v)\right) d r d s-\int f_{i} d \nu \int f_{k} d \nu\right|<M \gamma
$$


where $M$ is an upper bound for all the $f_{i}$ 's. Thus to get (1) our function $\varphi$ should have the property that on each interval of the form $\left[\frac{j}{N}, \frac{j+1}{N}\right]$, and most $t$ 's, the map $\{\varphi(z), \varphi(z+t)\}$ should take Lebesgue measure into a measure which is $\gamma$-close to the Lebesgue measure on the square $[0, T] \times[0, T]$ suitably normalized. It is clear that given $\varepsilon>0$ if $\gamma$ is small enough this will enable us to prove (i) with the original $\varepsilon$. After we accomplish this goal, (2) will be taken care of by a simple modification based on a Rohlin tower which is similar to what we did in $\S 1$.

We find it more convenient to work with a discrete situation so that we begin by finding some $L$ large enough so that for all $1 \leq i \leq N$ and all $u \in Y,\left|f_{i}\left(h_{s}(u)\right)-f_{i}(u)\right|<\frac{\gamma}{100 \cdot M}$ for all $|s| \leq \frac{100}{L}$. Now divide the square $[0, T] \times[0, T]$ into $L^{2}$-equal squares of size $\frac{1}{L} \times \frac{1}{L}$ and label them by andex set $A \times A$ with $A=\{1,2, \ldots, L\}$. The unit interval $[0,1]$ will now be divided into $n$ equal intervals of size $\frac{1}{n}$ each and we will construct $\varphi$ from a labelling of these intervals by indices from $A$ by having $\varphi$ vanish at the endpoints of each small interval of size $\frac{1}{n}$ and assume the value $\frac{a}{L} \cdot T$ on most of the interval where $a$ is the labelling. The equidistribution property that we want for $\varphi$ will follow from the following property for the sequence of labels $\left(a_{1}, a_{2}, \ldots, a_{n}\right)$ : There is a constant $q_{0}$ so that for any $q_{0} \leq q \leq n-q_{0}$ and any $m$, the pairs $\left(a_{m}, a_{m+q}\right),\left(a_{m+1}, a_{m+q+1}\right), \ldots,\left(a_{m+q_{0}}, a_{m+q+q_{0}}\right)$ are very well (to within $\left.\frac{\gamma}{100}\right)$ distributed over $A \times A$. Here we take $m+q$ etc., modulo $n$, since we are really on $\mathbb{R} / \mathbb{Z}$ rather than on $[0,1]$. While deterministic constructions can be given for sequences $a_{1}, \ldots, a_{n}$ with this kind of property it is somewhat easier to let chance do the work for us (although which is "easier" is surely a question of taste here).

Lemma. Given $\eta>0$, for all $n$ sufficiently large one can find sequences $a_{1} a_{2} \ldots$ $a_{n} \in A^{n}$ and a constant $q_{0}$ satisfying:

1. $q_{0} / n<\eta$.

2. for all $m \leq n, q_{0} \leq q \leq n-q_{0}$ we have

$$
\sum_{1 \leq i, j \leq L}\left|\frac{1}{L^{2}}-\frac{1}{q_{0}}\right|\left\{0<p \leq q_{0}:\left(a_{m+p}, q_{m+q+p}\right)=(i, j)\right\}||<\eta .
$$

(Here $|\{\cdot\}|$ denotes the number of elements in $\{\cdot\}$ and indices of $a_{m}$ are taken modulo $n$.)

Proof of lemma. We shall show that if we let the $a_{j}$ 's be independent random variables uniformly distributed on $\{1,2, \ldots, L\}$ then with high probability (*) will hold. For any fixed $q \geq q_{0}$ all the indices $\left\{m+p: 0<p \leq q_{0}\right\}$, $\left\{m+q+p: 0<p \leq q_{0}\right\}$ are disjoint so that the pairs of random variables $\left\{\left(a_{m+p}, a_{m+q+p}\right): 0<p \leq q_{0}\right\}$ are independent. It follows that there is a fixed constant $\lambda<1$ that depends only on $L$ and $\eta$ such that for fixed $m$ and $q,(*)$ holds with the exception of a set of probability at most $\lambda^{q_{0}}$. This follows from the standard exponential estimates of the tail for sums of independent identically distributed random variables (in our case Stirling's formula for the 
binomial distribution is adequate). This summing over the various possiblities for $m$ and $q$ gives us as an upper bound for the failure of at least one of the instances of $(*)$ the expression

$$
n^{2} \cdot \lambda^{q_{0}}=e^{2 \log n-(\log \lambda) q_{0}} .
$$

It remains to observe that for any large enough $n$,

$$
2 \log n-(\log \lambda) \cdot(\eta n / 10)<0
$$

and thus taking $q_{0}=[\eta n / 10]$ will guarantee that many (in fact most) randomly chosen sequences $a_{1} \cdots a_{n}$ will satisfy our requirements.

Choose parameters $\eta<\gamma$ small compared to $\frac{1}{N}$ and then use the sequence given by the lemma in the manner described above to define $\varphi$, periodic with period $\frac{1}{N}$, which will satisfy (1). At this point the set $D$ of good translations is basically all points that are not too close to one of the points $j / N$. In order that (2) hold, we need to get control over all points in the interval and so we need "Rohlin towers" that fill the entire space. Depending on the parity of $n$ with $p_{n} / q_{n}$ denoting the successive approximants to $\alpha$ in the continued fraction expansion, one gets the following "tower" picture of $\mathbb{R} / \mathbb{Z}$ with respect to $\alpha$ :

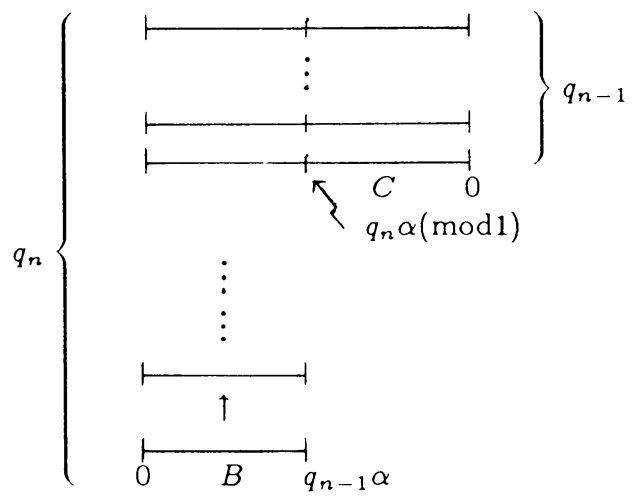

The transition from a point on one level to the point on the level immediately above it is given by adding $\alpha$ modulo 1 .

Since the two parts of the tower have unequal lengths and we wish to control the behavior of $\{\varphi(z), \varphi(z+t)\}$ for most $t$ 's, we have to introduce one more subdivision. Take $Q$ large and divide $C=\left[q_{n} \alpha, 0\right]$ into $Q$ equal pieces and then fill as much of $B=\left[0, q_{n-1} \alpha\right]$ as possible with contiguous intervals of the same length. Define $\tilde{\varphi}$ on $B \cup C$ by scaling the $\varphi$ defined above on each of these small intervals (all of equal size $=|C| / Q=d$ ) and extending identically up the columns to the whole of $[0,1]$. (On the part of $B$ left over $\tilde{\varphi}$ may be defined arbitrarily.) Note that $\tilde{\varphi}$ has two kinds of periodicity-

(i) it is almost invariant under translation by $\alpha$.

(ii) $[0,1]$ is almost filled up with intervals of the same length $d$ on each of which $\tilde{\varphi}$ is the same. 
Exploit (i) to define

$$
\varphi(z)=\frac{1}{K} \sum_{k=0}^{K-1} \tilde{\varphi}(z+k \alpha)
$$

with $K$ chosen so that $\frac{1}{K} \leq \frac{\delta}{10 \cdot T}$. This gives us (2) for all $z$. Furthermore, on most of the interval $\varphi$ agrees with $\tilde{\varphi}$ and (ii) still holds so that our previous estimates give (1). The bad sets of translations are basically numbers close to $p \alpha$ for $p<\left(q_{n}+q_{n-1}\right) \alpha$ or shifts of that by small multiples of $d$. In any event it is easily seen that for most $t$, the pair $\{\varphi(z), \varphi(z+t)\}$ is well distributed over the square $[0, T] \times[0, T]$. This completes the proof of Lemma 2.3.

\section{4. $\mathscr{P} \mathscr{J} \varsubsetneqq \mathscr{M}\left(\mathscr{W}^{\perp}\right)$ (TOPOLOGICAL CASE)}

A homomorphism $(X, T) \stackrel{\pi}{\rightarrow}(Y, T)$ of minimal flows is called regular if for every $\left(x_{1}, x_{2}\right) \in X \times X$ with $\pi\left(x_{1}\right)=\pi\left(x_{2}\right)$ there exists an automorphism $\psi$ of $(X, T)$ such that $\overline{\mathscr{O}}\left(x_{1}, x_{2}\right)$, the orbit closure of $\left(x_{1}, x_{2}\right)$, contains the graph $\Gamma_{\psi}=\{(x, \psi(x)): x \in X\}$. A joining of minimal flows $(X, T)$ and $(Y, T)$ is any closed invariant subset of $X \times Y .(X, T)$ and $(Y, T)$ are disjoint if $X \times Y$ is minimal. The following proposition is a slight generalization of Theorem 1 of [M]. When there is no room for confusion we omit the " $T$ " from the notation of a flow.

4.1 Proposition. Let $(X, T),\left(X_{1}, T\right),(Y, T)$ and $(Z, T)$ be minimal flows such that

(i) $X \stackrel{\pi}{\rightarrow} X_{1}$ is a regular homomorphism,

(ii) $Z$ is weakly mixing,

(iii) $X$ and $Y$ are disjoint from $Z$.

Then for any minimal joining $N$ of $X$ and $Y$, if $N_{1}=\{(\pi(x), y):(x, y) \in N\}$ is disjoint from $Z$ then so is $N$.

Proof. We choose a fixed minimal idempotent $u$ in $\beta \mathbb{Z}$ the Stone-Čech compactification of $\mathbb{Z}$. Let $W \subset N \times Z \subset X \times Y \times Z$ be a minimal joining of $N$ and $Z$. Choose $\left(x_{0}, y_{0}, z_{0}\right) \in W$ with $u\left(x_{0}, y_{0}, z_{0}\right)=\left(x_{0}, y_{0}, z_{0}\right)$. Since by assumption $N_{1} \times Z$ is minimal there exists a point $\left(x^{*}, y_{0}, T z_{0}\right) \in W$ with $\pi\left(x^{*}\right)=\pi\left(x_{0}\right)$; moreover, we can assume that $u x^{*}=x^{*}$. By regularity of $\pi$ there exists $\psi \in \operatorname{Aut}(X, T)$ such that $\psi x_{0}=x^{*}$.

Let $F$ be the cyclic group generated by the homeomorphism

$$
f: X \times Y \times Z \rightarrow X \times Y \times Z \text { where } f=\psi \times \operatorname{id} \times T .
$$

Since $(\psi \times \mathrm{id} \times T) W=W, W$ is $F$ invariant.

Let $\widetilde{X}$ be an $F$ minimal subset of $X$ then since $(Z, F)$ is weakly mixing, there exists a point $\left(x^{\prime}, z^{\prime}\right) \in \widetilde{X} \times Z$ with dense $F$ orbit. Choose $y^{\prime} \in Y$ with $\left(x^{\prime}, y^{\prime}, z^{\prime}\right) \in W$ (this is possible since $(X \times Z, T)$ is minimal). Then $W \supset \overline{F\left(x^{\prime}, y^{\prime}, z^{\prime}\right)} \supset\left(x^{\prime}, y^{\prime}\right) \times Z$. This however implies $W=X \times Y \times Z$ since $(X \times Y, T)$ is minimal. 
Let $\left(Y,\left\{h_{t}\right\}_{t \in \mathbb{R}}\right)=(Y, \mathbb{R})$ be a horocyclic flow on $Y=S L_{2}(\mathbb{R}) / \Gamma$ where $\Gamma$ is a uniform discrete subgroup of $S L_{2}(\mathbb{R})$. By [R] this flow is regular (i.e., a regular extension of the trivial one-point-flow). Thus given $y_{1}, y_{2} \in Y$ every minimal subset of the orbit closure $\operatorname{cls}\left\{h_{t}\left(y_{1}, y_{2}\right): t \in \mathbb{R}\right\}$ has the form $\Gamma_{\psi}=$ $\{(y, \psi(y)): y \in Y\}$ for some automorphism $\psi$ of $(Y, \mathbb{R})$.

4.2 Lemma. Let $(Z, \sigma)$ be an irrational rotation of the circle. Let $\left(Y,\left\{h_{t}\right\}_{t \in \mathbb{P}}\right)$ be the horocycle flow and put $X=Z \times Y$. If $\varphi: Z \rightarrow \mathbb{R}$ is a continuous map such that the corresponding homeomorphism: $T(z, y)=\left(\sigma z, h_{\varphi(z)}(y)\right)$ is minimal on $X$, then the homomorphism $(X, T) \stackrel{\pi}{\rightarrow}(Z, \sigma)$ is regular.

Proof. Let $\left(z_{0}, y_{1}\right),\left(z_{0}, y_{2}\right) \in X$, we will show that $\overline{\mathscr{O}}\left(\left(z_{0}, y_{1}\right),\left(z_{0}, y_{2}\right)\right)$ contains a graph

$$
\tilde{\Gamma}_{\psi}=\{((z, y)(z, \psi(y)): z \in Z, y \in Y\}
$$

for some automorphism $\psi$ of $(Y, \mathbb{R})$. Consider the sequence

$$
\varphi\left(n, z_{0}\right)=\varphi\left(\sigma^{n-1} z_{0}\right)+\varphi\left(\sigma^{n-2} z_{0}\right)+\cdots+\varphi\left(z_{0}\right) .
$$

If $\varphi(Z) \subset[-N, N] \subset \mathbb{R}$ then $\left\{\varphi\left(n, z_{0}\right)\right\}_{n \in \mathbb{Z}}$ is either bounded or syndetic with gaps $\leq 2 N$. If $\varphi\left(n, z_{0}\right)=\sum_{j=0}^{n-1} \varphi\left(\sigma^{j} z_{0}\right)$ is bounded then by [G-H, 14.11] $\varphi(z)=f(\sigma z)-f(z)$ for some continuous $f: Z \rightarrow \mathbb{R}$ in which case $(X, T)$ is not minimal contrary to our assumption. Thus $\left\{\varphi\left(n, z_{0}\right)\right\}_{n \in \mathbb{Z}}$ is syndetic and we can find a sequence $\left\{n_{i}\right\}$ such that $\sigma^{n_{i}} z_{0} \rightarrow z_{0}^{\prime}$ and $h_{\varphi\left(n_{i}, z_{0}\right)}\left(y_{1}, y_{2}\right) \rightarrow$ $\left(y_{1}^{\prime}, y_{2}^{\prime}\right)$ where the latter is an element of some minimal subset of $(Y \times Y, \mathbb{R})$. Since every such minimal set is of the form $\Gamma_{\psi}$ we have

$$
\begin{aligned}
T^{n_{i}}\left(\left(z_{0}, y_{1}\right),\left(z_{0}, y_{2}\right)\right) & =\left(\left(\sigma^{n_{i}} z_{0}, h_{\varphi\left(n_{1}, z_{0}\right.} y_{1}\right),\left(\sigma^{n_{i}} z_{0}, h_{\varphi\left(n_{1}, z_{0}\right)} y_{2}\right)\right) \\
& \rightarrow\left(\left(z_{0}^{\prime}, y_{1}^{\prime}\right)\left(z_{0}^{\prime}, y_{2}^{\prime}\right)\right)=\left(\left(z_{0}^{\prime}, y_{1}^{\prime}\right),\left(z_{0}^{\prime}, \psi\left(y_{1}^{\prime}\right)\right)\right)
\end{aligned}
$$

and

$$
\overline{\mathscr{O}}\left(\left(z_{0}, y_{1}\right)\left(z_{0}, y_{2}\right)\right) \supset \widetilde{\Gamma}_{\psi} .
$$

In the following corollaries $\mathscr{R}$ is the residual subset of $\overline{\mathscr{S}_{\mathscr{C}}(\sigma)}$ as in Theorem 2.2 (or Proposition 1.1 of $\left[\mathrm{G}_{1}\right]$ ).

4.3 Corollary. Let $(Z, \sigma)$ be an irrational rotation of the circle and let $(Y, \mathbb{R})$ be the horocycle flow. Then for each $T \in \mathscr{R}$ the homomorphism $\pi: X \rightarrow Z$ is regular.

4.4 Theorem. For $(Z, \sigma),(Y, \mathbb{R})$ as above and $T \in \mathscr{R},(X, T) \in \mathscr{M}\left(\mathscr{W}^{\perp}\right)$ and $(X, T) \notin \mathscr{P} \mathscr{F}$.

Proof. The first statement follows from Proposition 4.1 and Corollary 4.3. The second statement is proved in $\left[\mathrm{G}_{1}\right.$, Theorem 2.2].

Remark. As mentioned above the fact that $\mathscr{P} \mathscr{I} \subset \mathscr{M}\left(\mathscr{W}^{\perp}\right)$ follows by induction on the tower of proximal and isometric extensions of a given flow $(X, T)$ in $\mathscr{P} \mathscr{F}$. Another way of getting this fact is to use McMahon's theorem on 
the (nonmetric) universal $\mathscr{P} \mathscr{S}$-flow $\left|\mathfrak{A}\left(G_{\infty}\right)\right|$ which is regular (see e.g. $\left[\mathrm{G}_{3}\right]$ ). In fact if $(X, T)$ is any minimal regular flow then McMahon's theorem implies that $(X, T) \in \mathscr{M}\left(\mathscr{W}^{\perp}\right)$ iff $(X, T) \in \mathscr{W}^{\perp}$. Our extension of this theorem (Proposition 4.1) shows that $\mathscr{M}\left(\mathscr{W}^{\perp}\right)$ is closed under regular extensions in $\mathscr{W}^{\perp}$.

\section{REFERENCES}

[F] H. Furstenberg, Disjointness in ergodic theory, minimal sets, and a problem in diophantine approximation, Math. Systems Theory 1 (1967), 1-49.

[G 1 ] S. Glasner, Minimal skew products, Trans. Amer. Math. Soc. 260 (1980), 509-514.

$\left[\mathrm{G}_{2}\right]$ _, Quasi-factors in ergodic theory, Israel J. Math. 45 (1983), 198-208.

[G $\left.\mathrm{G}_{3}\right]$ _ Proximal flows, Lecture Notes in Math., vol. 517, Springer-Verlag, 1976.

[G-W] S. Glasner and B. Weiss, On the construction of minimal skew products, Israel J. Math. 34 (1979), 321-336.

[G-H] W. H. Gottschalk and G. A. Hedlund, Topological dynamics, Amer. Math. Soc. Colloq. Publ., Vol. 36, Amer. Math. Soc., Providence, R.I., 1955.

[M] D. McMahon, Multiple disjointness for weakly mixing regular minimal flows, Proc. Amer. Math. Soc. 98 (1986), 175-179.

[N] M. G. Nerurkar, Ergodic continuous skew product actions of amenable groups, Pacific J. Math. 119 (1985), 343-363.

[R] M. Ratner, Rigidity of horocycle flows, Ann. of Math. 115 (1982), 597-614.

[Z] R. J. Zimmer, Ergodic actions with generalized discrete spectrum. Illinois J. Math. 20 (1975), 555-588.

School of Mathematical Sciences, Tel Aviv University, Tel Aviv, Israel

Institute of Mathematics, The Hebrew University of Jerusalem, Jerusalem, IsRael 